\title{
Research on Improving Precision and Stability of Active Magnetic Levita- tion Feeding Guide System
}

\author{
Yanhong Zhang ${ }^{1,2, *}$, Dean Zhao ${ }^{1}$, Jiansheng Zhang ${ }^{2}$ and Zhongqiao Zheng ${ }^{2}$ \\ ${ }^{1}$ School of Electrical and Information Engineering, Jiangsu University, Zhenjiang, 212013, China \\ ${ }^{2}$ School of Electronic Information \& Electric Engineering, Changzhou Institute of Technology, Changzhou, 213002, \\ China
}

\begin{abstract}
The upscale CNC machine is the research object, the magnetic levitation bearing technology which is suitable to AMB spindle and guide are researched. On the basis of the structure and working principle of AMB and the stiffness and damping characteristics, the performance of electromechanical coupling dynamics is studied according to the theory of traditional dynamics and the characteristics of AMB. Then the theoretical calculation and simulation is made with several groups of different electrical parameters as input and the relationship between the controller parameters and the accuracy and stability of AMB feeding guide system, which provides a theoretical reference for achieving the optimal performance of magnetic levitation by adjusting the control parameters.
\end{abstract}

Keywords: Accuracy, controller, feeding guide, magnetic levitation, stability, stiffness and damping characteristics.

\section{INTRODUCTION}

With the progress of science and the development of technology, high speed cutting is developed rapidly in the machine manufacturing field of aerospace, automobile and motorcycle, mold and others. To some extent it has changed the status of metal cutting, greatly improved the efficiency of the metal cutting and surface precision, in order to realize high precision cutting. In addition to the system of high speed cutting tool, high speed and high precision of machine feeding system are two essential conditions as well [1-4]. The magnetic levitation guide technology of high-speed motorized spindle and high-speed linear guide are the main ways to realize high speed machine tools. Maglev supported technology is recognized as one of high and new technology in the world, which can be used as a high speed motorized spindle and high speed linear guide in high-grade $\mathrm{CNC}$ machine tool. Active Magnetic Bearing (AMB) has the advantages of no friction, no lubrication, environmentally safe, and long service life. It is being widely used in machine tools and high-speed rotating machinery, energy, transportation and other fields, especially in high-grade CNC machine with high speed. High-speed spindle and fast feed system are two key technologies to realize high speed process. The key problem is how to improve the processing speed and precision and stability of the feeding guide system by active magnetic suspension. This paper mainly studies the active magnetic suspension bearing technology of high-grade CNC machine, which on behalf of high speed and precision, is the theoretical and technological foundation of further research and development of motorized spindle and linear motors [5-9].

\section{STRUCTURE OF ACTIVE MAGNETIC LEVITA- TION FEEDING GUIDE SYSTEM}

Maglev feeding guide system is a device where a suspended feeder is guided by electromagnetic force and makes the guide movement by a linear motor. The bearing guide of feeding mechanism changes into contactless support from contact support by magnetic levitation technology, which makes the whole feed system frictionless and improves the feed precision of the system as well [10-12].

The maglev feeding guide system mainly includes electromagnet, feeding guide, bearing system, linear motor and control system. The structure of maglev feeding guide system is shown as Fig. (1). The air gap between feeding guide and guide is sent to controller, which is detected by displacement sensor, the controlled current or voltage signal from controller is magnified by an amplifier and sent to electromagnet coil, which makes magnetic field force change, so that the guide keeps a balance position.

The electromagnet is fixed on the feeding guide rail, the magnetic force from electromagnet makes feeding guide suspended and maintains a $0.2 \mathrm{~mm}$ suspension gap and the guiding electromagnetic iron provides enough horizontal magnetic force, which prevents them from contacting each other when the feed guide rail is subjected to the transverse force. Secondary coil of linear motor is installed under the feeding guide rail and the primary coil of linear motor is installed on linear motor. The secondary coil and the primary coil maintains a clearance of $0.5 \sim 0.8 \mathrm{~mm}$ between each other, the linear motor drives the secondary coil without any contact and the magnetic levitation guide makes controlled 


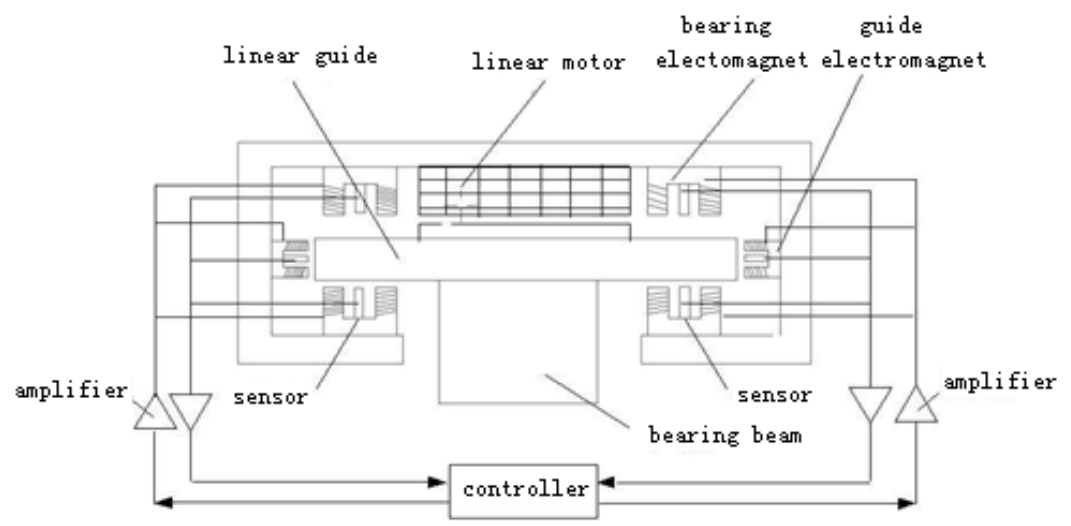

Fig. (1). Structure of active magnetic levitation feeding guide system.

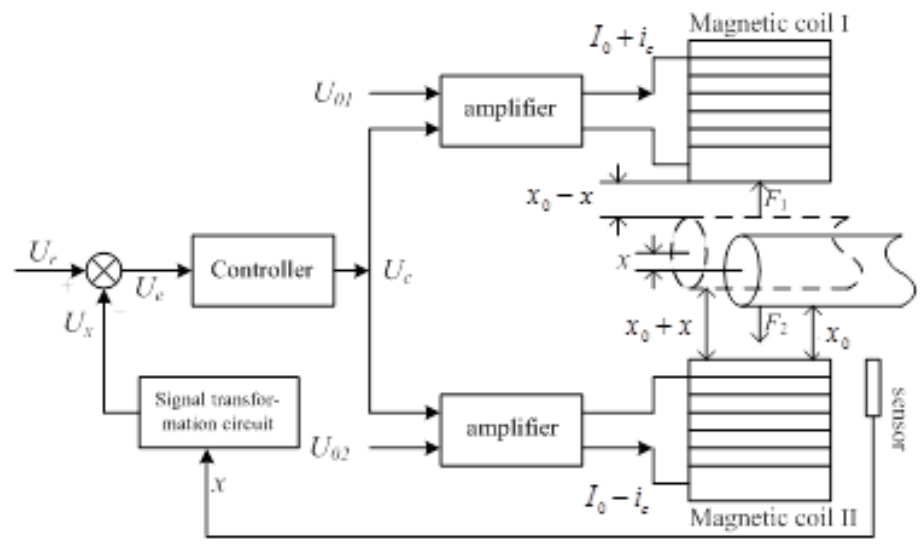

Fig. (2). Structure of active magnetic levitation system.

motion without friction accurately along the linear guide rail [13-17].

\section{MATHMATIC MODEL OF A SINGLE FREEDOM ACTIVE MAGNETIC LEVITATION}

A typical structure of single freedom active magnetic levitation system is mainly composed of electromagnet, displacement sensor, controller, power amplifier and rotors, which are shown in Fig. (2).

The following assumptions as well [16-18] are made in the active magnetic levitation system, which is shown as Fig. (1).

a. The rotor is considered as a single mass.

b. The hysteresis loss and eddy-current loss of magnetic materials are neglected.

c. The reluctance of core and rotor is neglected.

$\mathrm{d}$. The leakage flux of the winding is neglected.

Then the electromagnetic force on the rotor is

$$
\begin{aligned}
& F_{1}=\frac{\mu s_{0} N^{2}}{4}\left(\frac{I_{0}+i_{c}}{x_{0}+x}\right)^{2} \\
& F_{2}=\frac{\mu s_{0} N^{2}}{4}\left(\frac{I_{0}-i_{c}}{x_{0}-x}\right)^{2}
\end{aligned}
$$

Where,

$\mu$ - air permeability of ferromagnetic materials; $s_{0}$ - total pole face area; $N$ - number of turns per coil; $i_{c}$ - control current; $x_{0}$ - nominal air gap; $I_{0}$ - offset component of the current of the magnetic coil; $x$ - position displacement of the rotor center in the degree of freedom.

The motion equation of the rotor center of the radial bearing by electromagnetic force and an external force is

$$
m \ddot{x}=\Delta F=F_{1}-F_{2}=\frac{\mu s_{0} N^{2}}{4}\left[\left(\frac{I_{0}+i_{c}}{x_{0}+x}\right)^{2}-\left(\frac{I_{0}-i_{c}}{x_{0}-x}\right)^{2}\right]
$$

As $i_{c}<<I_{0}, x<<x_{0}$, neglecting quadratic term and higher order term of $i_{c}$ and $x$, the Taylor series expansion of the equation (3) can be obtained at the equilibrium point $x=0, i_{c}$ $=0$ as follows:

$$
m \ddot{x}=C_{1} x-C_{2} i_{c}
$$

Where,

$$
C_{1}=\frac{\mu s_{0} N^{2} I_{0}^{2}}{x_{0}^{3}} \text { - displacement stiff coefficient of active }
$$
magnetic bearing system

$$
C_{2}=\frac{\mu s_{0} N^{2} I_{0}}{x_{0}^{2}}-\text { current stiff coefficient of active magnet- }
$$

ic bearing system 


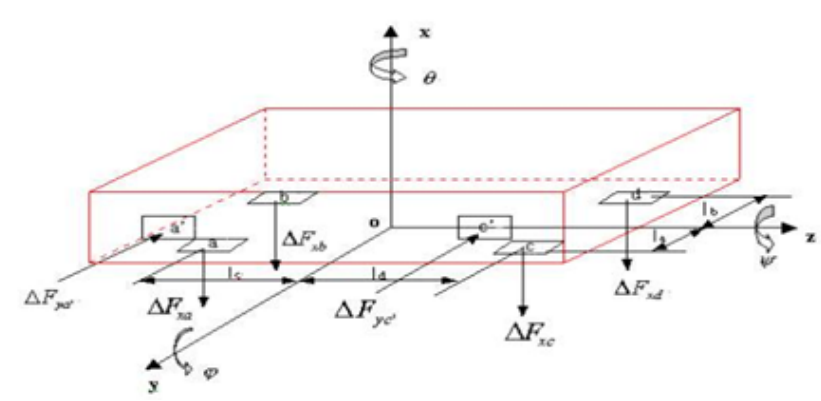

Fig. (3). The forced figure of five degree of freedom maglev feeding platform.

\section{THE MECHANICAL MODEL OF MAGNETIC LEVITATION FEEDING GUIDE}

The forced figure of five degree of freedom maglev feeding platform is shown as Fig. (3). The six degrees of freedom in space, five degrees of freedom are controlled by electromagnetic force, another degree of freedom which moves along $\mathrm{z}$ axis is driven by linear motor, therefore the system is a multivariable system. Take Cartesian coordinate origin as centroid o of guide, the coordinate of guide is shown as Fig. (3). Four parts of $a, b, c, d$ are equipped with electromagnets, the movement along $\mathrm{x}$ direction and rotation along $\mathrm{y}$ and $\mathrm{z}$ axis is controlled by electromagnetic force. Two parts of $a$, and $b^{\prime}$ are also equipped with electromagnets, the movement along y direction and rotation along $\mathrm{x}$ axis is controlled by electromagnetic force. Among them, the distance between a and $\mathrm{b}$ is $l_{a b}$, the distance between a and $\mathrm{c}$ is $l_{a c}$, the distance between a, c and o is $l_{a}$, the distance between $\mathrm{b}, \mathrm{d}$ and o is $l_{b}$, the distance between $\mathrm{a}, \mathrm{b}$ and $\mathrm{o}$ is $l_{c}$, the distance between $\mathrm{c}$, $\mathrm{d}$ and $\mathrm{o}$ is $l_{d}$, the guide slope angles are $\theta, \varphi, \psi$, the movement differential equations based on generalized coordinate are as follows.

$$
\left\{\begin{array}{l}
m \ddot{x}+\Delta F_{x a}+\Delta F_{x b}+\Delta F_{x c}+\Delta F_{x d}=0 \\
j_{y} \ddot{\phi}-\Delta F_{x a} L_{c}-\Delta F_{x b} L_{c}+\Delta F_{x c} L_{d}+\Delta F_{x d} L_{d}=0 \\
j_{z} \ddot{\psi}-\Delta F_{x a} L_{a}+\Delta F_{x b} L_{b}-\Delta F_{x c} L_{a}+\Delta F_{x d} L_{b}=0 \\
m \ddot{y}+\Delta F_{y a^{\prime}}+\Delta F_{y c^{\prime}}=0 \\
j_{x} \ddot{\theta}+\Delta F_{y a^{\prime}} L_{c}-\Delta F_{y c^{\prime}} L_{d}=0
\end{array}\right\}
$$

where $\mathrm{m}$ is the weight of guide rail, $j_{x}, j_{y}, j_{z}$ is rotational inertia of guide around the center of mass, $\mathrm{O}$ is the Barycentric coordinate of guide, $\theta$ for the vibration angular displacement around $\mathrm{x}$ axis, $\varphi$ for the vibration angular displacement around y axis, $l_{a}, l_{b}, l_{c}, l_{d}$ is the distance between four electromagnets and coordinate axis, $\Delta F_{x}$ and $\Delta F_{y}$ are the forces of guide along $\mathrm{x}$ and $\mathrm{y}$ axis, equation (6) is the linear equation of force, equation (7) is the coordinate transformation equation.

$$
\left\{\begin{array}{l}
\Delta F_{x a}=k_{x x}^{a} \cdot x_{a}+c_{x x}^{a} \cdot \dot{x}_{a} \\
\Delta F_{x b}=k_{x x}^{b} \cdot x_{b}+c_{x x}^{b} \cdot \dot{x}_{b} \\
\Delta F_{x c}=k_{x x}^{c} \cdot x_{c}+c_{x x}^{c} \cdot \dot{x}_{c} \\
\Delta F_{x d}=k_{x x}^{d} \cdot x_{d}+c_{x x}^{d} \cdot \dot{x}_{d} \\
\Delta F_{x a^{\prime}}=k_{x x}^{a^{\prime}} \cdot x_{a^{\prime}}+c_{x x}^{a^{\prime}} \cdot \dot{x}_{a^{\prime}} \\
\Delta F_{x b^{\prime}}=k_{x x}^{b^{\prime}} \cdot x_{b^{\prime}}+c_{x x}^{b^{\prime}} \cdot \dot{x}_{b^{\prime}}
\end{array}\right\}
$$

$$
\left\{\begin{array}{l}
x_{a}=x-l_{c} \phi-l_{a} \psi \\
x_{b}=x-l_{c} \phi+l_{b} \psi \\
x_{c}=x+l_{d} \phi-l_{a} \psi \\
x_{d}=x+l_{d} \phi+l_{b} \psi \\
y_{a^{\prime}}=y+l_{c} \theta \\
y_{c^{\prime}}=y-l_{d} \theta
\end{array}\right\}
$$

Take equation (7) and (6) into equation (5), the result is as follows.

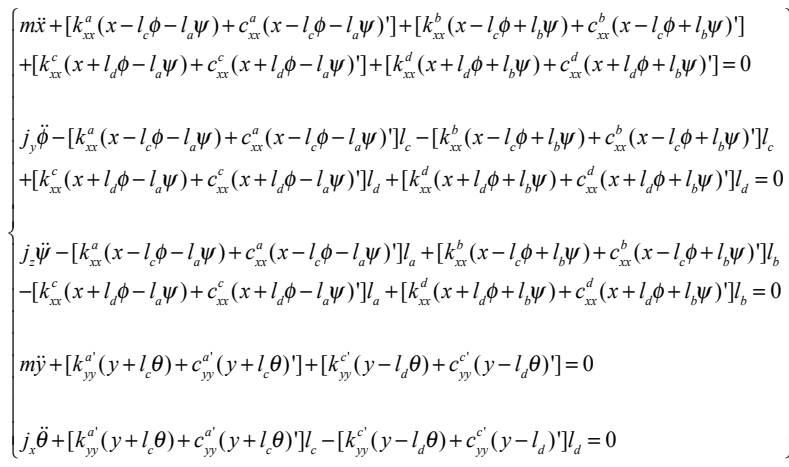

Then,

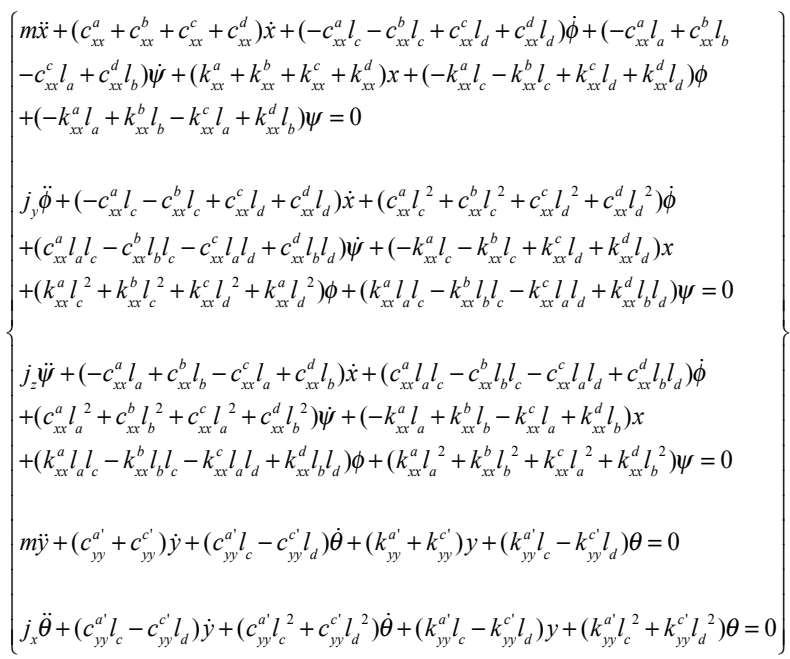

The motion differential equations based on matrix from equation (9) is follows.

$\bar{m} \ddot{q}+c \dot{q}+k q=0$

Where,

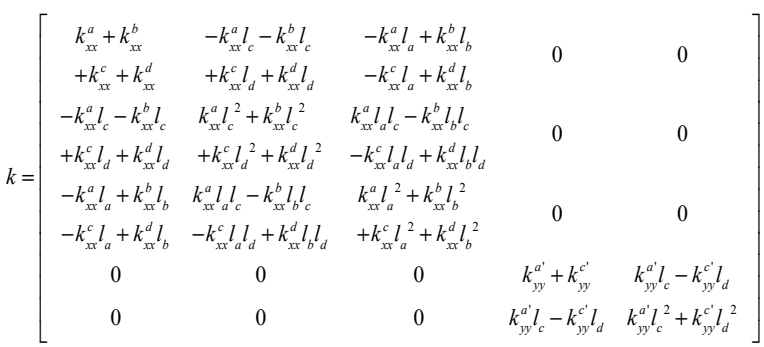




$$
\begin{aligned}
& q=\left[\begin{array}{l}
x \\
\phi \\
\psi \\
y \\
\theta
\end{array}\right] \\
& \bar{m}=\left[\begin{array}{ccccc}
m & 0 & 0 & 0 & 0 \\
0 & j_{y} & 0 & 0 & 0 \\
0 & 0 & j_{z} & 0 & 0 \\
0 & 0 & 0 & m & 0 \\
0 & 0 & 0 & 0 & j_{x}
\end{array}\right]
\end{aligned}
$$

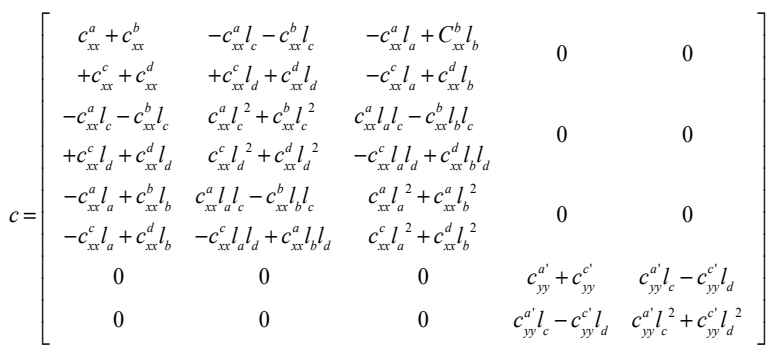

$q$ for the generalized displacement vector, $\bar{m}$ for a system of generalized mass matrix of system, $\mathrm{c}$ for generalized damping matrix of system, $k$ for generalized stiffness matrix of system.

Equation (10) is considered a coupling effect of guide electromagnet for lateral (i.e., Y direction) vibration, which is a five degrees of freedom differential equation of magnetic levitation feeding guide under a state of general eccentric mass. The eigenvalues of the maglev linear feeding guide system is gained by solving equation (10).

$$
\lambda_{r}=u+v i
$$

$u, v$ for real part and imaginary part of eigenvalue, the stability of the system can be seen from the eigenvalue real part, when the real part is negative, the system is stable, the imaginary part of eigenvalue is the system's inherent frequency.

In order to make the system analysis versatile and comparable and improve the stability and reliability of numerical calculation, it is necessary to make the system equations dimensionless parameters.

Dimensionless displacement

$$
\begin{aligned}
& X=\frac{x}{c_{0}}, Y=\frac{y}{c_{0}}, \Phi=\frac{l_{c d}}{2 c_{0}} \phi, \Psi=\frac{l_{a b}}{2 c_{0}} \psi, \Theta=\frac{l_{c d}}{2 c_{0}} \theta, L_{a}=\frac{2 l_{a}}{l_{a b}}, L_{b}=\frac{2 l_{b}}{l_{a b}} \\
& L_{c}=\frac{2 l_{c}}{l_{c d}}, L_{d}=\frac{2 l_{d}}{l_{c d}},
\end{aligned}
$$

\section{Dimensionless mass}

$$
M=\frac{m c_{0} \omega_{k}^{2}}{f_{m}}
$$

Dimensionless rotational inertia

$$
J_{x}=\frac{4 c_{0} \omega_{k}{ }^{2}}{f_{m} l_{c d}{ }^{2}} j_{x}, J_{y}=\frac{4 c_{0} \omega_{k}{ }^{2}}{f_{m} l_{c d}{ }^{2}} j_{y}, J_{z}=\frac{4 c_{0} \omega_{k}{ }^{2}}{f_{m} l_{c d}{ }^{2}} j_{z}
$$

Dimensionless damping and stiffness

$$
\begin{aligned}
& C_{x x}^{i}=\frac{c_{0} \omega_{k}}{f_{m}} c_{x x}^{i}, K_{x x}^{i}=\frac{c_{0}}{f_{m}} k_{x x}^{i},(i=a, b, c, d) \\
& C_{y y}^{i}=\frac{c_{0} \omega_{k}}{f_{m}} c_{y y}^{i}, K_{y y}^{i}=\frac{c_{0}}{f_{m}} k_{y y}^{i},\left(i=a^{\prime}, c^{\prime}\right)
\end{aligned}
$$

Other dimensionless parameters

$$
T=t \omega, \omega=\omega_{k} \Omega, \omega_{k}=\sqrt{\frac{g}{c_{0}}}, f_{m}=m g
$$

Take above dimensionless parameters into equation (9).

$$
\begin{aligned}
& J_{y} \Omega^{2} \ddot{\Phi}+\left(-C_{x x}^{a} L_{c}-C_{x x}^{b} L_{c}+C_{x x}^{c} L_{d}+C_{x x}^{d} L_{d}\right) \Omega \dot{X}+\left(C_{x x}^{a} L_{c}{ }^{2}+C_{x x}^{b} L_{c}{ }^{2}\right. \\
& \left.+C_{x x}^{c} L_{d}^{2}+C_{x x}^{d} L_{d}^{2}\right) \Omega \dot{\Phi}+\left(C_{x x}^{a} L_{a} L_{c}-C_{x x}^{b} L_{b} L_{c}-C_{x x}^{c} L_{a} L_{d}+C_{x x}^{d} L_{b} L_{d}\right) \Omega \dot{\Psi} \\
& +\left(-K_{x x}^{a} L_{c}-K_{x x}^{b} L_{c}+K_{x x}^{c} L_{d}+K_{x x}^{d} L_{d}\right) X+\left(K_{x x}^{a} L_{c}{ }^{2}+K_{x x}^{b} L_{c}{ }^{2}+K_{x x}^{c} L_{d}{ }^{2}+K_{x x}^{a} L_{d}{ }^{2}\right) \Phi \\
& +\left(K_{x x}^{a} L_{a} L_{c}-K_{x x}^{b} L_{b} L_{c}-K_{x x}^{c} L_{a} L_{d}+K_{x x}^{d} L_{b} L_{d}\right) \Psi=0 \\
& J_{z} \Omega^{2} \ddot{\Psi}+\left(-C_{x x}^{a} L_{a}+C_{x x}^{b} L_{b}-C_{x x}^{c} L_{a}+C_{x x}^{d} L_{b}\right) \Omega \dot{X}+\left(C_{x x}^{a} L_{a} L_{c}-C_{x x}^{b} L_{b} L_{c}\right. \\
& \left.-C_{x x}^{c} L_{a} L_{d}+C_{x x}^{d} L_{b} L_{d}\right) \Omega \dot{\Phi}+\left(C_{x x}^{a} L_{a}^{2}+C_{x x}^{b} L_{b}{ }^{2}+C_{x x}^{c} L_{a}^{2}+C_{x x}^{d} L_{b}^{2}\right) \Omega \dot{\Psi} \\
& +\left(-K_{x x}^{a} L_{a}+K_{x x}^{b} L_{b}-K_{x x}^{c} L_{a}+K_{x x}^{d} L_{b}\right) X+\left(K_{x x}^{a} L_{a} L_{c}-K_{x x}^{b} L_{b} L_{c}-K_{x x}^{c} L_{a} L_{d}+K_{x x}^{d} L_{b} L_{d}\right) \Phi \\
& +\left(K_{x x}^{a} L_{a}^{2}+K_{x x}^{b} L_{b}{ }^{2}+K_{x x}^{c} L_{a}^{2}+K_{x x}^{d} L_{b}^{2}\right) \psi=0 \\
& M \Omega^{2} \ddot{Y}+\left(C_{y y}^{a^{\prime}}+C_{y y}^{c^{\prime}}\right) \Omega \dot{Y}+\left(C_{y y}^{a^{\prime}} L_{c}-C_{y y}^{c^{\prime}} L_{d}\right) \Omega \dot{\Theta}+\left(K_{y y}^{a^{\prime}}+K_{y y}^{c^{\prime}}\right) Y+\left(K_{y y}^{a^{\prime}} L_{c}-K_{y y}^{c^{\prime}} L_{d}\right) \Theta=0 \\
& J_{x} \Omega^{2} \ddot{\Theta}+\left(C_{y y}^{a^{\prime}} L_{c}-C_{y y}^{c^{\prime}} L_{d}\right) \Omega \dot{Y}+\left(C_{y y}^{a^{\prime}} L_{c}{ }^{2}+C_{y y}^{c^{\prime}} L_{d}{ }^{2}\right) \Omega \dot{\Theta}+ \\
& \left(K_{y y}^{a^{\prime}} L_{c}-K_{y y}^{c^{\prime}} L_{d}\right) Y+\left(K_{y y}^{a^{\prime}} L_{c}{ }^{2}+K_{y y}^{c^{\prime}} L_{d}{ }^{2}\right) \Theta=0
\end{aligned}
$$

Dimensionless matrix type for above equation is follows.

$$
\bar{M} Q^{\prime \prime}+C Q^{\prime}+K Q=0
$$

Where,

$Q=\left[\begin{array}{c}X \\ \Phi \\ \Psi \\ Y \\ \Theta\end{array}\right], \bar{M}=\left[\begin{array}{ccccc}M \Omega^{2} & 0 & 0 & 0 & 0 \\ 0 & J_{y} \Omega^{2} & 0 & 0 & 0 \\ 0 & 0 & J_{z} \Omega^{2} & 0 & 0 \\ 0 & 0 & 0 & M \Omega^{2} & 0 \\ 0 & 0 & 0 & 0 & J_{x} \Omega^{2}\end{array}\right]$

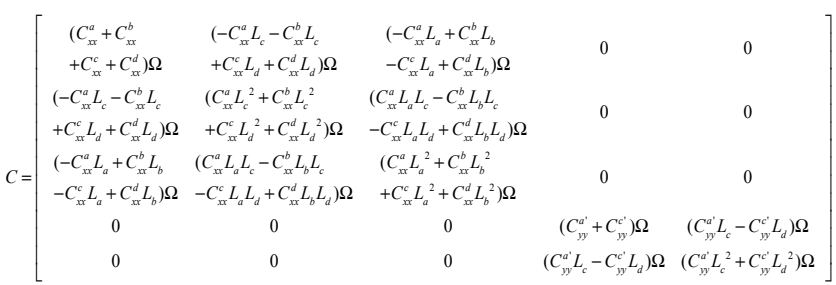

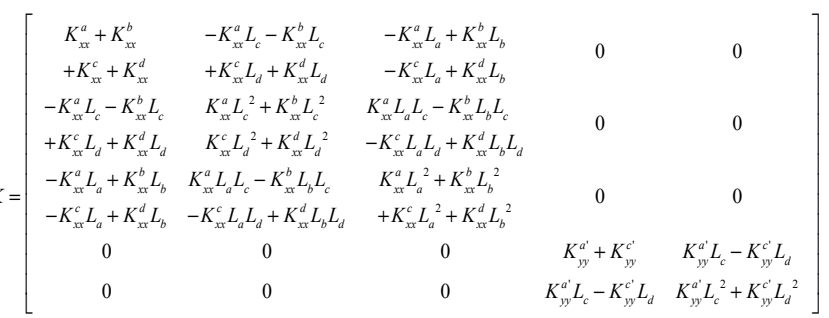


Table 1. The parameter of controller.

\begin{tabular}{|c|c|c|c|c|}
\hline Channel & $\boldsymbol{W}_{\boldsymbol{s}} \boldsymbol{k} \boldsymbol{\Omega}$ & $\boldsymbol{W}_{\boldsymbol{p}} \boldsymbol{k} \boldsymbol{\Omega}$ & $\boldsymbol{W}_{\boldsymbol{i}} \boldsymbol{k} \boldsymbol{\Omega}$ & $\boldsymbol{W}_{\boldsymbol{d}} \boldsymbol{k} \boldsymbol{\Omega}$ \\
\hline \hline 1 & 4.79 & 0.350 & 2.72 & 1.62 \\
\hline 2 & 3.44 & 0.290 & 3.18 & 1.46 \\
\hline 3 & 3.80 & 0.442 & 3.17 & 1.87 \\
\hline 4 & 4.56 & 0.354 & 1.98 & 1.59 \\
\hline 5 & 2.60 & 0.380 & 2.58 & 1.72 \\
\hline
\end{tabular}

$M, C$ and $K$ is the generalized non-dimensional mass matrix, damping matrix and stiffness matrix of the system, respectively. Q is the generalized non dimensional displacement vector. The corresponding forced vibration equation is follows.

$$
\bar{M} Q^{\prime \prime}+C Q^{\prime}+K Q=F
$$

Where, $\mathrm{F}$ is forced vibration vector, which is composed by external interference force.

\section{STATE EQUATION OF SYSTEM}

Because there is a damping matrix $\mathrm{C}$ in the vibration differential equation (18), the equation can't be decoupled by regular equation in an un-damped system. Although $\mathrm{M}, \mathrm{K}$ can become diagonal matrix after coordinate transformation, but $\mathrm{C}$ cannot usually become diagonal matrix, so the equation cannot be decoupled. The characteristic value and characteristic vector appeared in the vibration differential equation (18), the state space method is used in this paper.

Introduction of state vector, $Y=\left[Q^{\prime}, Q\right]$

The state equation corresponding to the movement differential equation (18) is as follows.

$\left[\begin{array}{cc}C & M \\ M & 0\end{array}\right] Y^{\prime}+\left[\begin{array}{cc}K & 0 \\ 0 & -M\end{array}\right] Y=0$

if

$B=\left[\begin{array}{cc}C & M \\ M & 0\end{array}\right], A=-\left[\begin{array}{cc}K & 0 \\ 0 & -M\end{array}\right]$

Then the state equation is shortened as follows.

$B Y^{\prime}=A Y$

The state equation (19) is a first order differential equation. It can be seen that the differential equation can be reduce an order when the state vector $\mathrm{Y}$ is introduced by state space method.

Equation (18) is solved by Matlab program [19], the eigenvalue of maglev linear feeding guide system can be obtained in any work time.

$\lambda_{r}=U+V i$
Where, $U=u / \omega$ and $V=v / \omega$, when $\mathrm{V}$ is close to 1 , the corresponding critical vibration frequency is known.

The every order critical frequency of system can be obtained, the corresponding logarithmic decrement rate for:

$\delta_{r}=-2 \pi \operatorname{Re}\left(\lambda_{r}\right) / \operatorname{Im}\left(\lambda_{r}\right)$

The order critical frequency whose logarithmic decrement rate is negative is the resonance instability frequency of the system.

\section{THE PERFORMANCE CALCULATION AND ANALYSIS}

There are some parameters of the Maglev linear feeding guide system in this paper, the mass of feeding guide is 49.8 $\mathrm{kg}$, four pairs of electromagnets are adopted in radial direction, Two pairs of electromagnets are in the transverse direction, sectional area of supporting electromagnet is $0.0009 \mathrm{~m}^{2}$, sectional area of guide electromagnet is $0.0004 \mathrm{~m}^{2}$. The number of coils are 240 , the bias current is $1 \mathrm{~A}$, the radial and transverse air gap is both $0.2 \mathrm{~mm}$.

The following dynamic performance calculations of feeding guide is done under ideal conditions (i.e. $\left.x_{a 0}=x_{b 0}=x_{c 0}=x_{d 0}=0, y_{a}{ }^{\prime} 0=y_{c^{\prime} 0}=0\right)$. The dynamic performance is gained by setting parameter of controller, Table 1 shoes the parameter of controller. The corresponding main vibration mode is shown in Fig. (4).

Table 2 shows the critical vibration frequency and the logarithmic decrement rate of the system, Fig. (5). is the corresponding stiffness and damping curve.

It can be seen from the figures above that there are five orders of critical vibration frequency. The logarithmic decrement rate corresponding to the first and second order critical vibration frequency is very big, which shows the stability margin of the system is large enough. The resonance happens when the feeding guide passes the two critical frequencies, the resonance amplitude is not too big because of the bearing damping. It can be seen from Fig. (6). that the real part of eigenvalue is less than zero, which can well meet the requirements of stability, the real part of eigenvalue become positive until $1662 \mathrm{~Hz}$, it shows that the resonance instability frequency is $1662 \mathrm{~Hz}$, so there is a close relationship between the instability of system and bearing damping. The logarithmic decrement rate corresponding to the latter $3^{\text {rd }}$ 


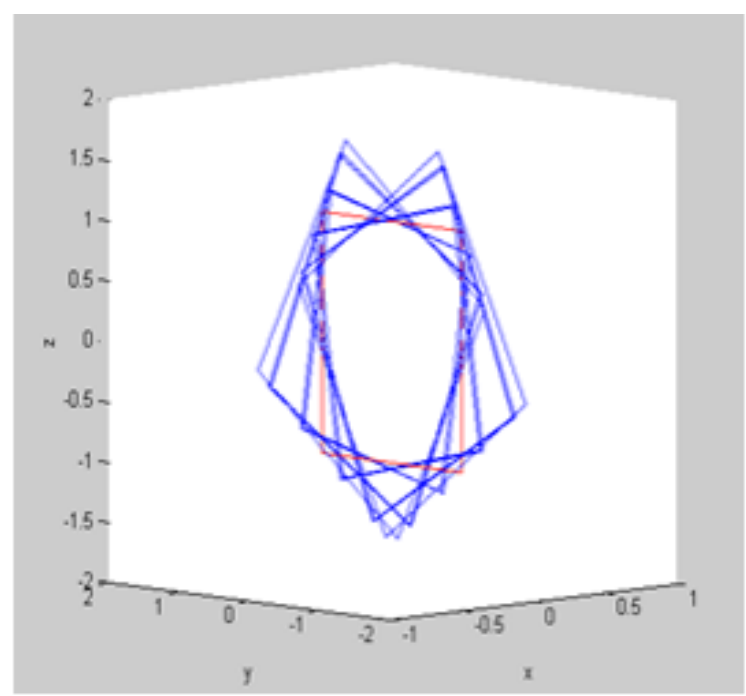

one order main vibration type

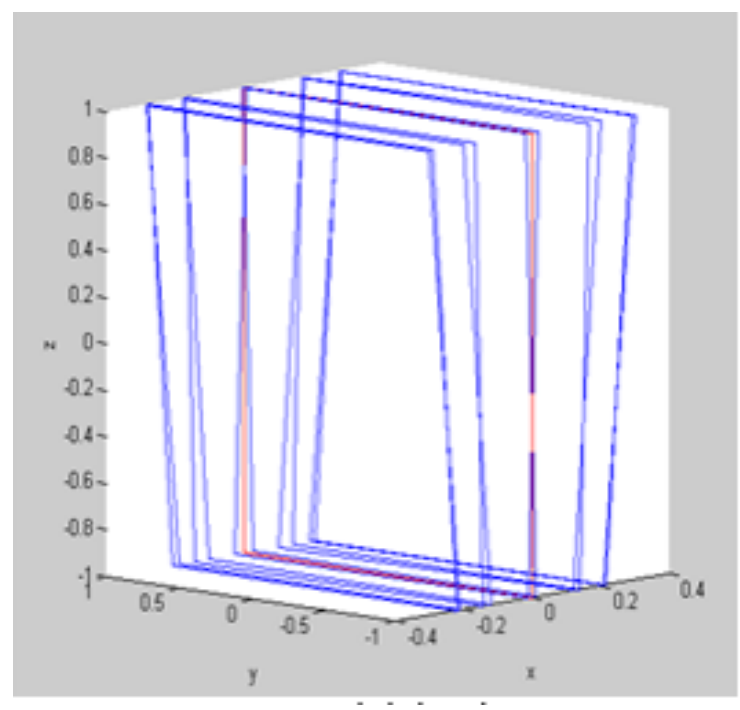

two order main vibration type

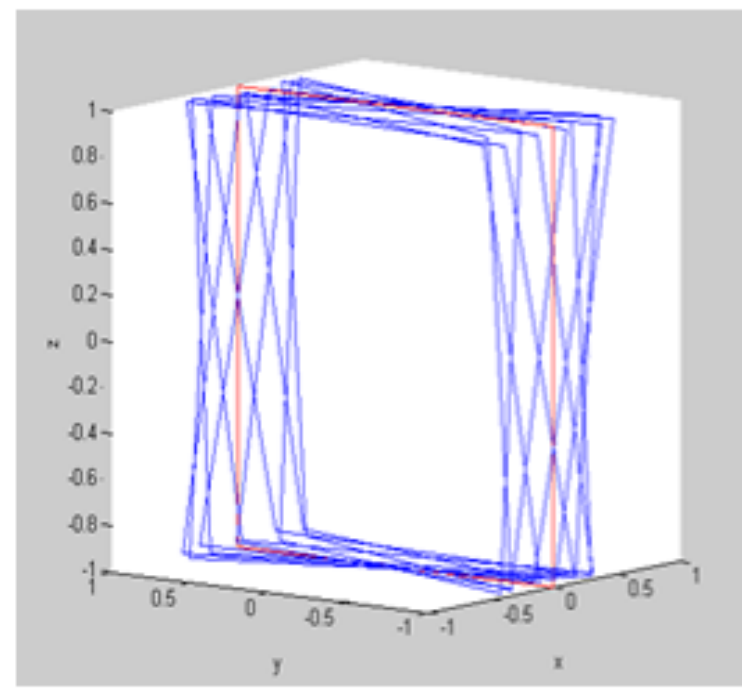

three order main vibration type

Fig. (4). contd... 


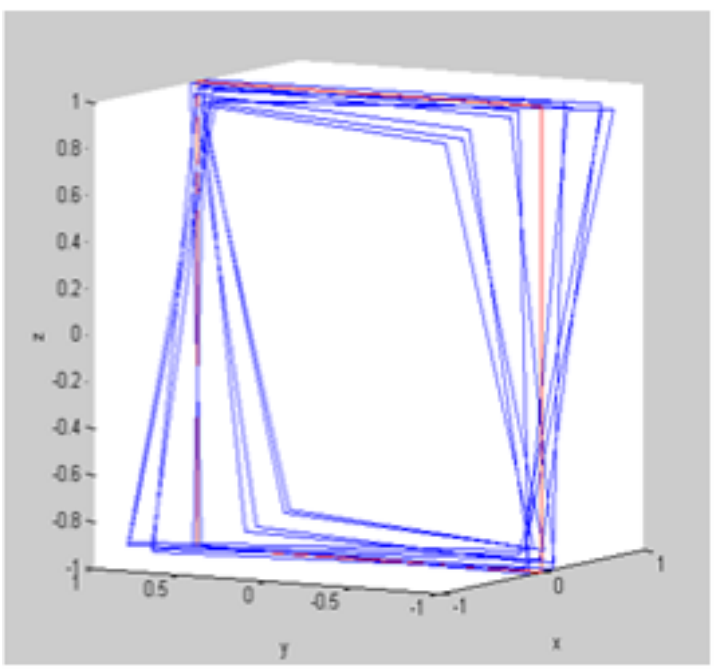

four order main vibration type

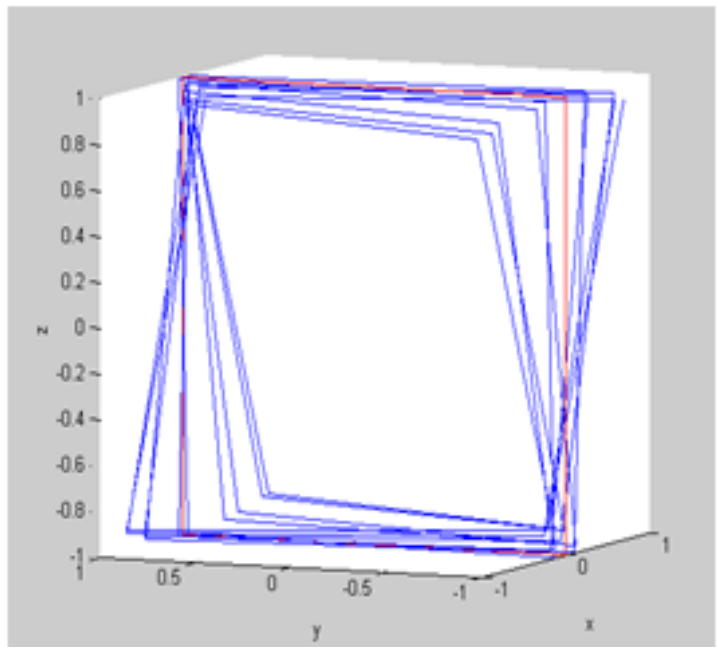

five order main vibration type

Fig. (4). Main vibration type of every order.

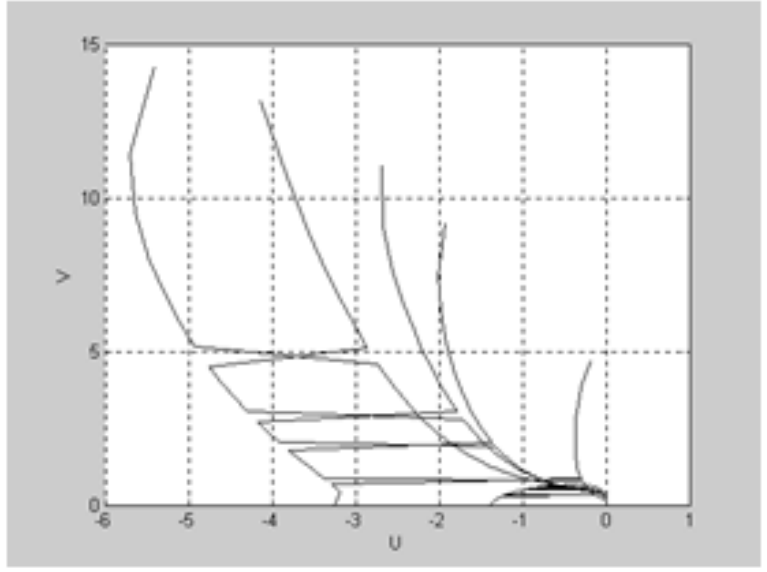

Fig. (5). Eigenvalue trajectory. 
Table 2. The critical vibration frequency and decrement rate.

\begin{tabular}{|c|c|c|}
\hline & the Inherent Frequency $(\mathbf{H z})$ & The Logarithmic Decrement Rate \\
\hline \hline One order & 40.18 & 3.1842 \\
\hline Two order & 44.75 & 3.4661 \\
\hline Three order & 64.96 & 0.9101 \\
\hline Four order & 75.74 & 0.9224 \\
\hline Five order & 83.35 & 1.1401 \\
\hline Frequency of resonance instability type $(\mathrm{Hz})$ & & 1662 \\
\hline
\end{tabular}
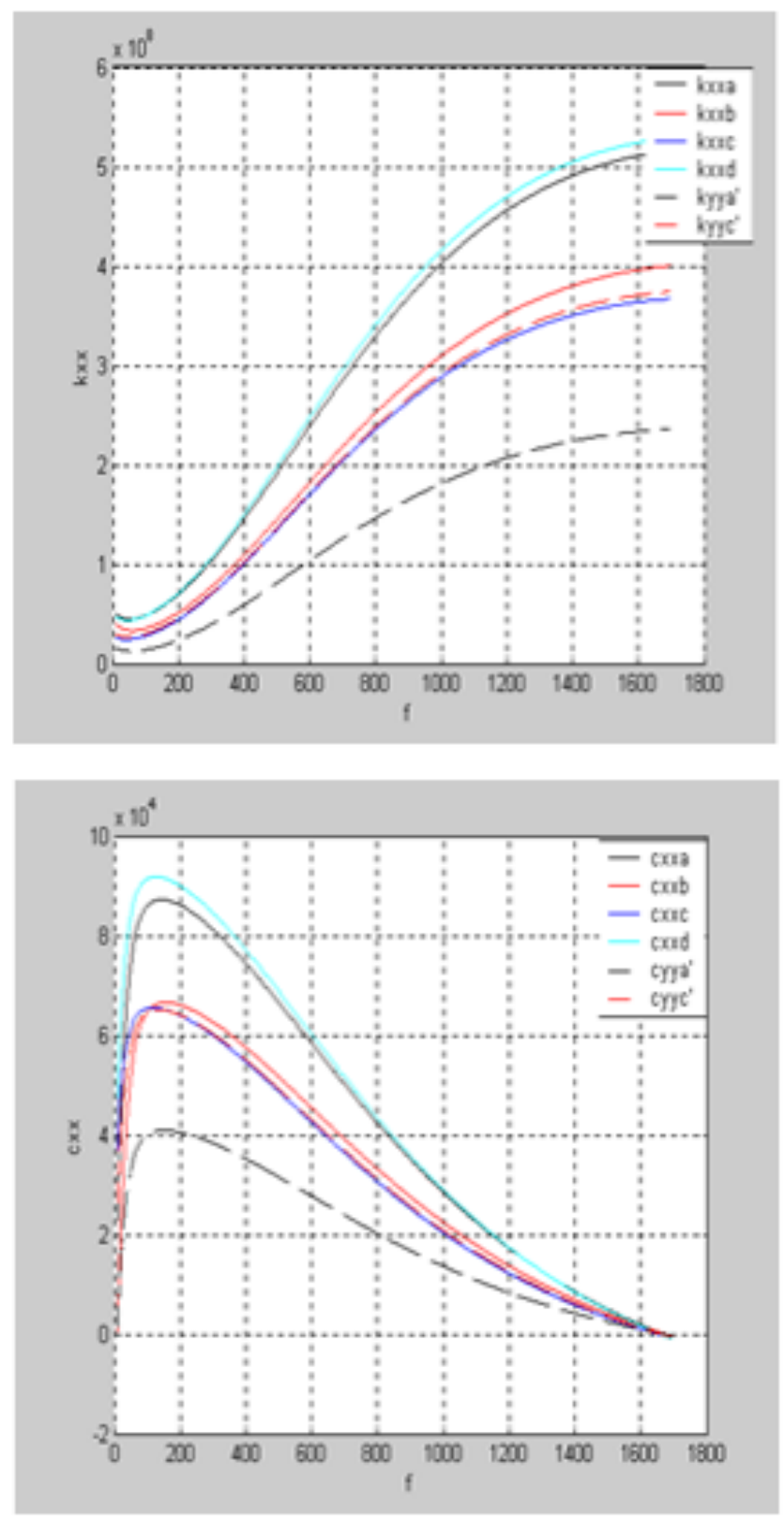

Fig. (6). The corresponding stiffness and damping curve.

order critical vibration frequency is around 1 , which also meets the requirements of engineering. From Fig. (4), we found that the interference between the lateral support and the radial bearing is small. The movement mode of one order 
critical vibration swing mainly in $\mathrm{y}_{\mathrm{oz}}$ plane, there is almost movement in the $\mathrm{x}$ direction. The movement mode of two order critical vibration swing mainly in $\mathrm{x}$ direction up and down, which is similar to the rigid body and there is almost movement in the y direction. The movement mode of three order critical vibration swing rotating about $\mathrm{y}$ axis and in $\mathrm{x}$ direction up and down, there is almost movement in the $y$ direction. The fourth order and fivth order main vibration is also in the $\mathrm{x}$ direction on the swing, but it is different from third order as it swings up and down from two diagonal points.

\section{CONCLUSION}

To sum up, the stiffness damping borne by the electromagnetic is not only associated with the structural parameters of itself, but also by the parameters of the controller. Several channel controls determines that there are several different stiffness and damping characteristics curves in the system, the real part of eigenvalue must be negative if the system is to be stable. The largest vibration amplitude happened in the installation of the electromagnet, the vibration amplitude is smaller in the center of feeding guide, which is important for feeding guide, because all kinds of components such as a clamp, spindle, etc. are installed at the center of feeding guide. The processing accuracy can be improved if the vibration amplitude is small, so that the performance of magnetic levitation feeding guide system can be optimum by setting appropriate parameters.

\section{CONFLICT OF INTEREST}

The authors confirm that this article content has no conflict of interest.

\section{ACKNOWLEDGEMENTS}

This work was supported by the National Natural Science Foundation of China (Grant No. 51175052), the basic application and research program of Changzhou(Grant No. CJ20130014), the scientific research fund project of Changzhou Institute of Technology (Grant No.YN1216).

\section{REFERENCES}

[1] R. Zimbelman, "A Contribution to the Problem of CementAggregate Bond", Cement and Concrete Research, vol. 15, pp. 801-808, June 1985.

[2] G. Schweitzer, H. Bleuler, and A. Traxler, "Basics, Properties and Applications of Active Magnetic Bearings", New Times Press, Beijing, 1994.
[3] K. Chang-Hyun, K. Ki-Jung, Y. Ju-Seong and C. Han-Wook, "Dynamic Performance Evaluation of 5-DOF Magnetic Levitation and Guidance Device by Using Equivalent Magnetic Circuit Model", IEEE Transactions on Magnetics, vol. 49, No. 7, pp. 4156-4159, 2013.

[4] J. Zhang, X. Mei, D. Zhang, G. Jiang, and Q. Liu, "Application of decoupling fuzzy sliding mode control with active disturbance rejection for MIMO magnetic levitation system", Proceedings of the Institution of Mechanical Engineers, vol. 227, No. 2, pp. 213-229, 2013.

[5] W. Jiang, and H. B. Zhao, "Variable Structure Control for Active Magnetic Bearings", Proceeding of the $5^{\text {th }}$ International Symposium on Magnetic Bearings, vol. 39, pp. 215-219, 1996.

[6] J.C. Doyle, "Feedback Control Theory", TsingHua University Press, Beijing, 1993.

[7] T. Namerikawa, M. Fujita, and F. Matsumura, "Wide Area Stabilization of a Magnetic Bearing Using Exact Linearization", Proceeding of the 6th International Symposium on Magnetic Bearings, vol. 23, pp. 733-742, 1998.

[8] S. Masahiko, S. Ryunosuke, and S. Toshihiko, "Nonlinear resonances of three modes in a high-T c superconducting magnetic levitation system", Physica C: Superconductivity and its Applications, vol. 494, pp. 265-269, 2013.

[9] W. Min, and G. Weihua, "Modem Robust Control", Central South University of Technology Press, Changsha, 1998.

[10] W. Zhou, and B. B. Liu, "Backstepping Based Adaptive Control of Magnetic Llevitation System", Applied Mechanics and Materials, vol. 341, pp. 945-948, 2013.

[11] D. L. Trumper, S. M. Olson, and P. K. Subrahmanyan, "Linearizing Control of Magnetic Suspension Systems", IEEE Trans on Control Systems Technology, vol. 5, pp. 427-437, 1997.

[12] H. B. Yang, S. J. Yu, R. J. Yang, Y. H. Dai, and C. S. Tang, "A New Hybrid Excited Magnetic Levitation Feeding Platform", $A p$ plied Mechanics and Material, vol.385, pp. 772-776, 2013.

[13] F. Matsumura, T. Namerikawa, K. Hagiwara, and M. Fujita, "Application of Gain Scheduled $\mathrm{H} \infty$ Robust Controllers to a Magnetic Bearing", IEEE Trans on Control Systems Technology, vol. 4, pp. 484-493, 1996.

[14] Y. Kuruma, A. Yamamoto, and T. Higuchi, "High Speed NonContact Object Handling Using Magnetic Levitation and Tilt Control", Applied Mechanics and Materials, vol. 162, pp. 471-476, 2012.

[15] S. Jianliang, P. Yan, and L. Hongmin, "Dynamic Modeling of Gauge Control System Based on Thickness Gauge and Design of $\mathrm{H}$ $\infty$ Robust Controller", Journal of Mechanical Engineering, vol. 45, No. 6, pp. 160-170, 2009.

[16] W. Wiboonjaroen, and S. Sujitjorn, "State-PID Feedback for Magnetic Levitation System", Advanced Materials Research, No. 622, pp. 1467-1473, 2013.

[17] L. Qunming, Z. Ling, and X. Zhen, "Robust controller design of maglev ball system", Journal of Central South University (Science and Technology), vol. 38, No. 5, pp. 922-927, 2007.

[18] J. Baranowski, "Observer-based feedback for the magnetic levitation system", Transactions of the Institute of Measurement and Control, vol. 34, No. 4, pp. 422-435, 2012.

[19] Xue Dingyu, "Design and Analysis of Feedback Control SystemMATLAB Language Application", TsingHua University Press, Beijing, 2000.

Received: September 22, 2014

Revised: November 01, 2014

Accepted: November 05, 2014

(C) Zhang et al.; Licensee Bentham Open.

This is an open access article licensed under the terms of the Creative Commons Attribution Non-Commercial License (http://creativecommons.org/licenses/by-nc/3.0/) which permits unrestricted, non-commercial use, distribution and reproduction in any medium, provided the work is properly cited. 